\title{
Resolving vorticity and dissipation in a turbulent boundary layer by tomographic PTV and VIC+
}

\author{
Jan F. G. Schneiders ${ }^{1} \mathbb{D} \cdot$ Fulvio Scarano $^{1} \cdot$ Gerrit E. Elsinga $^{2}$
}

Received: 12 October 2016 / Revised: 21 January 2017 / Accepted: 6 February 2017 / Published online: 18 March 2017

(C) The Author(s) 2017. This article is published with open access at Springerlink.com

\begin{abstract}
The existing time-resolved tomographic particle image velocimetry (PIV) measurements by Jodai and Elsinga (J Fluid Mech 795:611-633; Jodai, Elsinga, J Fluid Mech 795:611-633, 2016) in a turbulent boundary layer $\left(R e_{\theta}=2038\right)$ are reprocessed using tomographic particle tracking velocimetry (PTV) and vortex-in-cellplus (VIC+). The resulting small-scale flow properties, i.e. vorticity and turbulence dissipation, are compared. The $\mathrm{VIC}+$ technique was recently proposed and uses the concept of pouring time into space to increase reconstruction quality of instantaneous velocity. The tomographic PTV particle track measurements are interpolated using VIC+ to a dense grid, making use of both particle velocity and Lagrangian acceleration. Comparison of the vortical structures by visualization of isosurfaces of vorticity magnitude shows that the two methods return similar coherent vortical structures, but their strength in terms of vorticity magnitude is increased when using VIC + , which suggests an improvement in spatial resolution. Further statistical evaluation shows that the root mean square (rms) of vorticity fluctuations from tomographic PIV is approximately $40 \%$ lower in comparison to a reference profile available from a DNS simulation, while the VIC+ technique returns rms vorticity fluctuations to within $10 \%$ of the reference. The
\end{abstract}

Electronic supplementary material The online version of this article (doi:10.1007/s00348-017-2318-x) contains supplementary material, which is available to authorized users.

Jan F. G. Schneiders

J.F.G.Schneiders@tudelft.nl

1 Department of Aerospace Engineering, TU Delft, Delft, The Netherlands

2 Department of Mechanical, Maritime and Materials Engineering, TU Delft, Delft, The Netherlands dissipation rate is heavily underestimated by tomographic PIV with approximately 50\% damping, whereas the VIC+ analysis yields a dissipation rate to within approximately $5 \%$ for $y^{+}>25$. The fact that dissipation can be directly measured by a volumetric experiment is novel. It differs from existing approaches that involve $2 \mathrm{~d}$ measurements combined with isotropic turbulence assumptions or apply corrections based on sub-grid scale turbulence modelling. Finally, the study quantifies the spatial response of VIC+ with a sine-wave lattice analysis. The results indicate a twofold increase of spatial resolution with respect to cross-correlation interrogation.

\section{Introduction}

Following two decades of experimental and numerical work in wall-bounded turbulent flows, as reviewed in Marusic et al. (2010), today's experiments by tomographic particle image velocimetry (PIV) have allowed for unprecedented volumetric and time-resolved measurement of such flows (Schröder et al. 2008, 2011; Elsinga and Marusic 2010; Elsinga et al. 2012). Recent studies have, however, stumbled upon the spatial resolution limitations of tomographic PIV for measurements in a turbulent flow (Atkinson et al. 2011; Kähler et al. 2012; Lynch et al. 2014). Velocity fluctuations are typically dominated by large-scale events, which can be resolved even with a relatively coarse spatial resolution. On the other hand, the components of the velocity gradient tensor, which govern vorticity and energy dissipation, act at smaller scale and thus require a higher spatial resolution. For the turbulent dissipation, a vector spacing of 2 Kolmogorov length scales is recommended (Tokgoz et al. 2012). This point is illustrated in Fig. 1 for a turbulent boundary layer, where the velocity fluctuations 
Fig. 1 Wall normal profiles of the rms of the fluctuating velocity (a) and vorticity (b) components. Time-resolved tomographic PIV measurement of Jodai and Elsinga (2016) (solid lines) and DNS data of Schlatter and Örlü (2010) (dashed lines) are presented. The $x, y$ and $z$ coordinates correspond to streamwise, wallnormal and spanwise directions, respectively
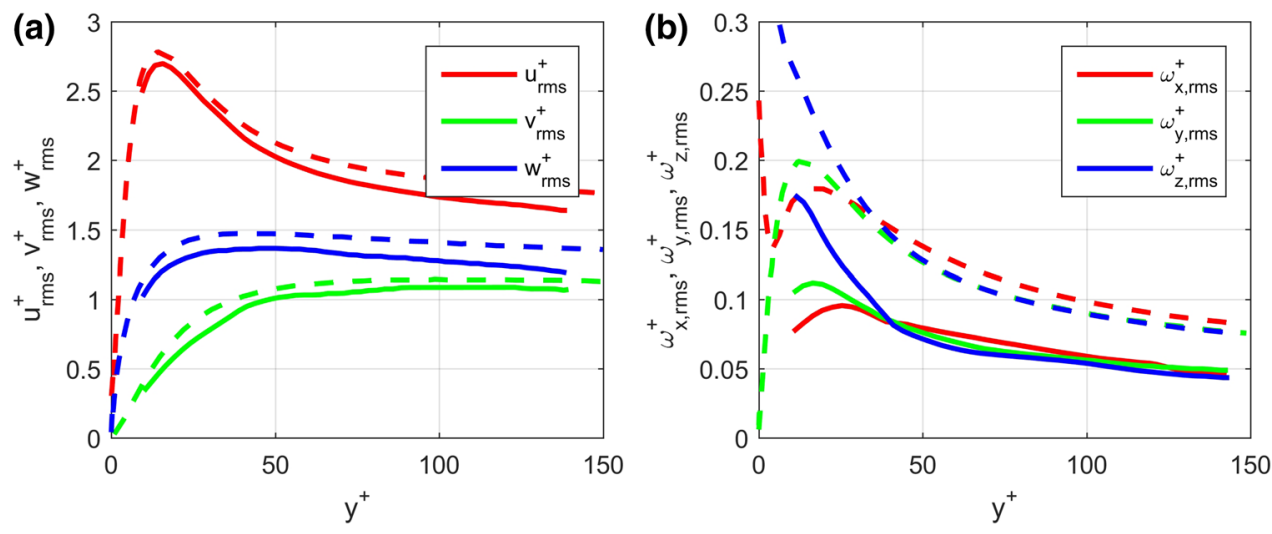

measured by time-resolved tomographic PIV agree with direct numerical simulation (DNS) data to within 5\%. The root mean square (rms) vorticity profiles, instead, are systematically underestimated to approximately half the value predicted by DNS. The spatial resolution in these measurements thus captures the velocity fluctuations accurately, but at the same time under-resolves the (peak) vorticity fluctuations. For velocity statistics, high spatial resolution can be achieved by ensemble particle tracking velocimetry (ensemble PTV) approaches as proposed by Kähler et al. (2012) and refined for volumetric experiments by Agüera et al. (2016). This approach evaluates velocity statistics in small interrogation volumes from the full PTV velocity measurement time series. For evaluation of statistics that are dependent on instantaneous spatial velocity gradients (e.g. vorticity, strain rate, turbulent dissipation), the ensemble PTV approach cannot be used and one needs to use techniques that are based on the evaluation of instantaneous gradients from PTV measurements.

Using time-resolved Lagrangian particle tracking in tomographic reconstructions (i.e. tomographic PTV) to increase the dynamic range of instantaneous velocity and acceleration measurements was explored by Novara and Scarano (2013). In the latter work, the fluid flow acceleration was interpolated from the scattered particle positions onto a Cartesian mesh by a polynomial regression. Recently, the Shake-the-Box algorithm (STB, Schanz et al. 2016) was introduced to perform Lagrangian particle tracking in densely seeded flows based on the iterative particle reconstruction technique (IPR, Wieneke 2013) instead of tomographic reconstruction. Particle tracking techniques yield results at the scattered instantaneous particle positions, which have motivated the development of advanced interpolators to obtain measurements on a regular grid. The use of flow governing equations to increase measurement accuracy was explored by Zhong et al. (1991) and Vedula and Adrian (2005), who included the incompressibility constraint. In the case where the temporal resolution is limited, instead of spatial resolution, simulation of the flow governing equations in vorticity-velocity formulation through the vortex-in-cell (VIC) technique has successfully been applied to tomographic PIV measurements to increase the temporal resolution (pouring space into time, Schneiders et al. 2014, 2016). Recently, this idea was reversed and the VIC technique was used also to leverage temporal information from time-resolved tomographic PIV measurements for noise reduction in Schneiders et al. (2015). Moreover, when dealing with time-resolved particle tracking (e.g. from tomographic PTV or 'Shake-the-Box'), the recently proposed vortex-in-cell-plus (VIC+) technique (Schneiders and Scarano 2016) interpolates velocity from the scattered particle locations onto a fine grid. The dense interpolation is made possible using not only the instantaneous velocity measurements, but also including the instantaneous particle acceleration. The measurements are linked to instantaneous velocity on the grid using both the continuity equation and the vorticity transport equation. The above approach was shown to significantly increase the accuracy of the Reynolds stresses in comparison to cross-correlation-based analysis used for tomographic PIV and other interpolation techniques used for particle tracking velocimetry. Also the recently proposed FlowFit method (Gesemann et al. 2016) shows increased measurement accuracy when using both velocity and instantaneous acceleration measurements. Furthermore, in a collaborative work conducted within the European NIOPLEX consortium (van Gent et al. 2017) several methods were compared making use of a synthetic experiment based on a numerical simulation. It was found that the spatial interpolation of sparse data, when conducted with the VIC+ approach, yields a very accurate reconstruction of the instantaneous pressure field compared to alternative techniques. It remains to be demonstrated whether similar improvements are achieved also for statistics of quantities dependent on the velocity gradient tensor (e.g. vorticity and dissipation) in actual experiments, where factors such as calibration errors, image noise and ghost particles are present (Elsinga et al. 2011). 
In the present manuscript, the VIC+ technique is applied to an actual experiment in a turbulent boundary layer, in order to assess the accuracy of the velocity gradients, vorticity and dissipation. For the present analysis, the existing experimental data of Jodai and Elsinga (2016) are used. These authors have performed time-resolved tomographic PIV measurements to obtain a detailed characterization of the turbulent motions in a turbulent boundary layer. The experiments are conducted in the same conditions as the DNS study of Schlatter and Örlü (2010). The comparison showed good agreement with the reference in terms of velocity statistics (Fig. 1a). Instead, vorticity fluctuations are systematically underestimated (Fig. 1b), which is ascribed to the limited spatial resolution of the measurement. In the present work, the VIC+ technique is applied to these measurements with the aim of assessing the potential benefits when evaluating properties dominated by small-scale motions, such as vorticity and dissipation. In addition, the spatial response and resolution of the VIC + technique are quantified by means of an analytical sine-wave lattice analysis in the Appendix. The Appendix is included to support the spatial resolution increase observed in the turbulent boundary layer experiment, and provides reproducible results for assessment of methods developed in the future.

\section{Experimental setup}

The time-resolved tomographic PIV turbulent boundary layer experiment was performed in a water channel at the TU Delft. A full description of the experiment is given by Jodai and Elsinga (2016). Relevant details related to the boundary layer properties and measurement setup are repeated here for completeness. The Reynolds number based on the momentum thickness, $R e_{\theta}$, and the friction velocity, $R e_{\tau}$, were 2038 and 782, respectively. The free-stream velocity, $U_{\infty}$, was $0.253 \mathrm{~m} / \mathrm{s}$, while the boundary layer thickness, $\delta_{99}$, was $69.9 \mathrm{~mm}$. Since the paper focusses on the near wall region and small-scale quantities, the relevant turbulent scales for comparison are the so-called wall units, which are summarized in Table 1 . Here, $v$ is the kinematic viscosity and $u_{\tau}$ is the friction velocity. Normalization by these wall units is indicated by the superscript +

The measurement volume spanned 60 and $55 \mathrm{~mm}$ in the streamwise $(x)$ and spanwise $(z)$ direction, respectively, while it covered $13 \mathrm{~mm}$ in the wall-normal $(y)$ direction starting at the wall $(y=0)$. The tracer particles were illumined by a high-speed laser, and their images were recorded by four

Table 1 Turbulent boundary layer scales used for normalization cameras. The recording rate equalled $1279 \mathrm{~Hz}$, resulting in approximately 3.3 pixels maximum particle displacement between frames. In total, three time-series of 3140 images were recorded, corresponding to $7.37 \mathrm{~s}$ of observation time. The present temporal resolution causes subsequent time samples to be highly correlated. Correlated samples, however, do not contribute to statistical convergence. So for reason of computational efficiency, the data are analysed in bursts for tomographic PTV processing with VIC+. Each burst is a short sequence of $24 \mathrm{~ms}$ on which particle tracking analysis is performed. This analysis yields for each burst the velocity and acceleration of the tracer particles, allowing VIC+ analysis to obtain the instantaneous velocity fields. The time separation between bursts is chosen at $0.2 \mathrm{~s}$, corresponding to 0.7 large-scale turnover times $\left(\delta_{99} / U_{\infty}\right)$. The flow properties are homogeneous along the span and are considered homogeneous along the streamwise direction over the length of the measurement domain. The data ensemble along both directions is considered for the statistical evaluation of the turbulent properties. For further details of the experimental setup, the reader is referred to Jodai and Elsinga (2016).

\section{Data processing methods}

The recorded images were originally processed using tomographic PIV as outlined in Sect. 3.1. In the present work, the data are evaluated using VIC+ and the processing steps are outlined in Sect. 3.2. For both tomographic PIV and VIC+ processing, the same set of recorded images is used.

\subsection{Tomographic PIV}

Tomographic PIV processing was performed by Jodai and Elsinga (2016) and salient aspects are recalled in this section. The intensity volumes were reconstructed using the MART algorithm (Elsinga et al. 2006) and the average intensity profile of the reconstruction in wall-normal direction as calculated from the reconstructed objects is reported in Fig. 2a, which is used to obtain the wall location in the reconstructed volumes. The intensity profile shows at $y^{+}=$ 0 a sharp intensity increase. Towards the end of the illuminated volume, a more gradual reduction in intensity is found up to $y^{+} \approx 150$, after which the intensity decreases to the noise level. The sharp change in intensity at $y^{+}=0$ indicates the wall location, which is obtained at an accuracy on the order of the particle image diameter in the reconstructed volumes, which equals 3 voxels (1.4 wall units).

The particle displacement field was obtained from the sequence of reconstructed volumes using direct cross-correlation. Object pairs were taken with a time separation of $4.6 \mathrm{~ms}$ (factor six sub-sampling) yielding a maximum particle displacement of 19.8 voxels, which enhances the dynamic 
Fig. 2 Wall normal profiles of reconstructed light intensity by MART (a) and density of detected particle tracks (b)

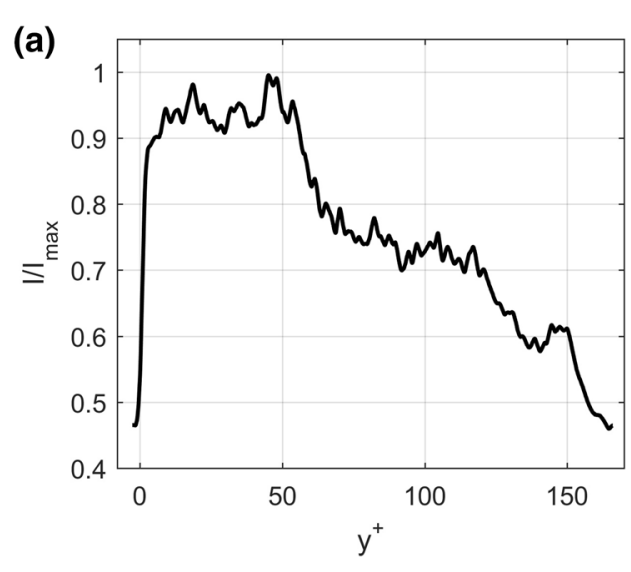

velocity range. The first reconstructed volume was thus crosscorrelated with the 7 th volume in the time sequence, the 2nd with the 8th volume and so on. The resulting temporal oversampling of the velocity field was used later to further suppress measurement noise. The final size of the interrogation volume was $32 \times 32 \times 32$ voxels $\left(1.37 \times 1.37 \times 1.37 \mathrm{~mm}^{3}\right)$ and neighbouring interrogation volumes overlap by $75 \%$. The resulting vector spacing in each direction was $0.34 \mathrm{~mm}$ (approx. 3.8 wall units). The region near the wall $\left(y^{+}<40\right)$ was also processed using a final correlation window of $64 \times 16 \times 32$ voxels at $75 \%$ overlap to improve the spatial resolution along the wall-normal direction. The universal outlier detection method was used to remove spurious vectors (Westerweel and Scarano 2005). To suppress measurement noise, the velocity fields were spatiotemporally filtered using a second-order polynomial regression over a period of $14.1 \mathrm{~ms}$ $\left(1.7 \mathrm{v} / u_{\tau}^{2}\right)$ and in a cubic filter volume of $2.05^{3} \mathrm{~mm}^{3}$, which corresponds to $23^{3}$ wall units. In the near-wall region, the filter volume was $4.10 \mathrm{~mm} \times 1.02 \mathrm{~mm} \times 2.05 \mathrm{~mm}(46 \times 12 \times 23$ wall units) due to the different interrogation volume used. The coefficients of the polynomial regression were also used to determine the local value of the velocity gradients.

\subsection{Tomographic PTV with VIC+}

The VIC+ method (Schneiders and Scarano 2016) interpolates scattered PTV measurements towards a Cartesian grid. The method is briefly recalled below. It considers PTV measurements of instantaneous particle velocity, $\boldsymbol{u}_{\mathrm{PTV}}$, and its Lagrangian temporal derivative, D $\boldsymbol{u}_{\mathrm{PTV}} / \mathrm{D} t$. The VIC+ method iteratively seeks for the vorticity field that minimizes a cost function. This cost function considers the difference between the measured data and the result generated with VIC + , which is obtained by solution of the vorticity transport equation,

$\frac{\mathrm{D} \boldsymbol{\omega}}{\mathrm{D} t}=\frac{\partial \boldsymbol{\omega}}{\partial t}+(\boldsymbol{u} \cdot \nabla) \boldsymbol{\omega}=(\boldsymbol{\omega} \cdot \nabla) \boldsymbol{u}$,

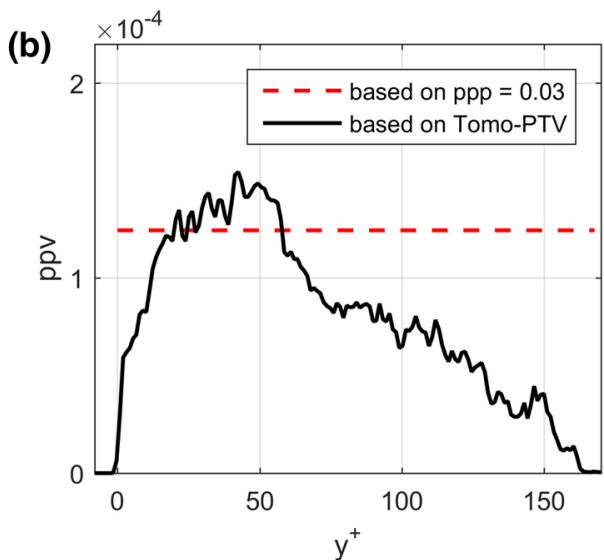

where velocity is related to vorticity through a Poisson equation,

$\nabla^{2} \boldsymbol{u}=-\nabla \times \boldsymbol{\omega}$,

Specific details of the present tomographic PTV and VIC+ processing are given in the remainder of this section. For implementation details and a detailed pseudo-code of the VIC+ method, the reader is referred to Schneiders and Scarano (2016). In addition to the analysis of the turbulent boundary layer, the present manuscript also quantifies the frequency response of the VIC+ method in comparison to tomographic PIV (given in the Appendix).

Particle trajectories are obtained using the same tomographic PTV algorithm as reported in Schneiders and Scarano (2016). This algorithm uses the volumetric intensity distribution as obtained with sequential motion tracking enhanced MART (SMTE, Lynch and Scarano 2015). The reconstruction is applied to the measurement timeseries sub-sampled by a factor two, yielding a maximum particle displacement of 6.6 voxels. Particle locations are identified with sub-voxel accuracy using a 3-point Gaussian fit along each coordinate direction. A particle-tracking algorithm based on Malik et al. (1993) is used to find particle tracks and the minimum acceleration criterion is used in case multiple particles are identified within a search box. The tomographic PIV results (Sect. 3.1) are used as predictor for the tracking algorithm. The discrete positions of a particle in motion are then used to produce a least-squares 3rd order polynomial fit estimating the trajectory over 15 exposures. The result yields the fitted particle position at each time instant. Its time derivative yields the velocity and in turn the Lagrangian acceleration (viz. velocity material derivative). Figure $2 b$ shows the profile of identified particles per voxel (ppv) along wall-normal direction following from the PTV algorithm (black line). The water tunnel was seeded homogeneously and, therefore, a uniform track density is expected at an estimated level of 0.0013 particles per voxel (ppv, dashed red line), based on an estimate 
of 0.03 particles per pixel (ppp) from the recorded images. For $y^{+}>60$, a lower number of particles is observed, which is associated with lower laser light intensity reducing particle detectability. The estimated ppv based on the particle image density, therefore, represents a volume average concentration of detected particles. The actual seeding concentration can be higher, and will be closer to the peak ppv in Fig. 2b.

After particle tracking, the iterative VIC+ procedure is started with the results obtained from tomographic PTV. The no-slip condition is prescribed at the wall. The VIC+ method discretizes the vorticity field using Gaussian radial basis functions. Schneiders and Scarano (2016) indicate a base function spacing, $h$, for VIC+ on the order of $h=0.25$ $\mathrm{ppv}^{-1 / 3}$. Following this criterion, in the present study the spacing of the base functions is set to $0.25 \mathrm{~mm}$, corresponding to 2.8 wall units, for $y^{+}<60$. Further away from the wall less tracks are identified and hence a coarser base function spacing of $0.5 \mathrm{~mm}$ (5.6 wall units) is used. The computational grid is evaluated at a factor two oversampling resulting in a vector spacing of $0.125 \mathrm{~mm}$ (1.4 wall units) in the near-wall region and $0.25 \mathrm{~mm}$ ( 2.8 wall units) for $y^{+}>60$. All gradients are obtained using second-order finite differences on the computational grid.

\section{Results and discussion}

The validity of the velocity reconstructions is first examined by analysis of the velocity statistics (Sect. 4.1). Subsequently, the instantaneous flow organization is studied (Sect. 4.2) and the statistics of vorticity fluctuations are considered (Sect. 4.3). The discussion concludes with the estimation of the dissipation rate (Sect. 4.4).

\subsection{Velocity statistics}

The velocity statistics are compared to the results by Schlatter and Örlü (2010) from a DNS simulation at $R e_{\theta}=$ 2000 , which is close to the Reynolds number in the experiment $\left(R e_{\theta}=2038\right)$. The performance of tomographic PIV using the cubic and elongated interrogation volumes for the measurement of the velocity statistics in this experiment is already discussed in Jodai and Elsinga (2016). They are recalled here for comparison to the results from VIC+. The mean velocity profile along wall-normal direction is plotted in Fig. 3. The blue and yellow lines show the results obtained by tomographic PIV with, respectively, the cubic and elongated interrogation volumes. The red line shows the VIC+ result. For reference, the black line shows the DNS results. For $10<y^{+}<140$, all methods agree well with each other, indicating that the time averaged velocity profile is captured by all approaches and that the experiments agree with the DNS data.

The PIV result with cubic interrogation volumes of $32 \times 32 \times 32$ voxels shows its first vector at 16 voxels, corresponding to 7.6 wall units. With elongated correlation volumes, the first vector that results from interrogation windows not overlapping with the wall is located at 8 voxels (3.8 wall units) from the wall. Vectors below this wallnormal distance result from interrogation volumes overlapping with the wall and overestimate the mean velocity. The result of VIC+ is in good correspondence to the PIV results for $y^{+}>3$. Closer to the wall it follows the DNS reference profile and because the no-slip condition is prescribed at the wall for VIC+ the velocity goes exactly to zero at the wall.

The profiles of rms velocity fluctuations are given in Fig. $4 \mathrm{a}-\mathrm{c}$, for the streamwise, wall-normal and spanwise components of velocity, respectively. The PIV results with both cubic and elongated interrogation windows are largely equivalent, with the exception that the near wall peak at $y^{+}=15$ in the streamwise velocity fluctuations (Fig. 4a) is captured slightly better by tomographic PIV with the elongated volumes. The VIC+ estimate of the streamwise velocity fluctuations (Fig. 4a) follows closely the PIV results, which match well with the DNS result for $y^{+}<50$. Instead, the streamwise fluctuations depart from the DNS data for $y^{+}>50$ with approximately $10 \%$ lower values. The latter can be related to the slightly favourable pressure gradient in the water channel facility and the uncertainty in determining $u_{\tau}$ (Jodai and Elsinga 2016). For the wall-normal and spanwise velocity fluctuations (Fig. 4b, c, respectively), tomographic PIV shows slightly lower values than the DNS result along the full $y$-range, but remains within $10 \%$ between $y^{+}=50$ and 140. The VIC+ analysis follows more closely the DNS (within 5\%) for the wall-normal (Fig. 4b) and spanwise (Fig. 4c) components. This behaviour already indicates a potential improvement in rendering turbulent fluctuations with the VIC+ analysis.

Because divergence of velocity is expected to be zero in an incompressible flow, recent studies have used the level of velocity divergence to assess the measurement accuracy of the velocity gradient (de Silva et al. 2013; Jodai and Elsinga 2016; amongst others). The velocity divergence consists of three components,

$\nabla \cdot \boldsymbol{u}=\frac{\partial u}{\partial x}+\frac{\partial v}{\partial y}+\frac{\partial w}{\partial z}$

The joint probability density function of $-\partial u / \partial x$ and $(\partial v / \partial y+\partial w / \partial z)$ is plotted in Fig. 5. Points away from the diagonal correspond to non-zero divergence. The comparison of the results obtained from tomographic PIV (a) and VIC+ (b) indicates a significantly lower divergence error for VIC+. The calculation of the cross-correlation 
Fig. 3 Time-averaged velocity profiles returned by tomographic PIV with cubic and elongated correlation volumes and with VIC+, in comparison to the DNS result by Schlatter and Örlü (2010)

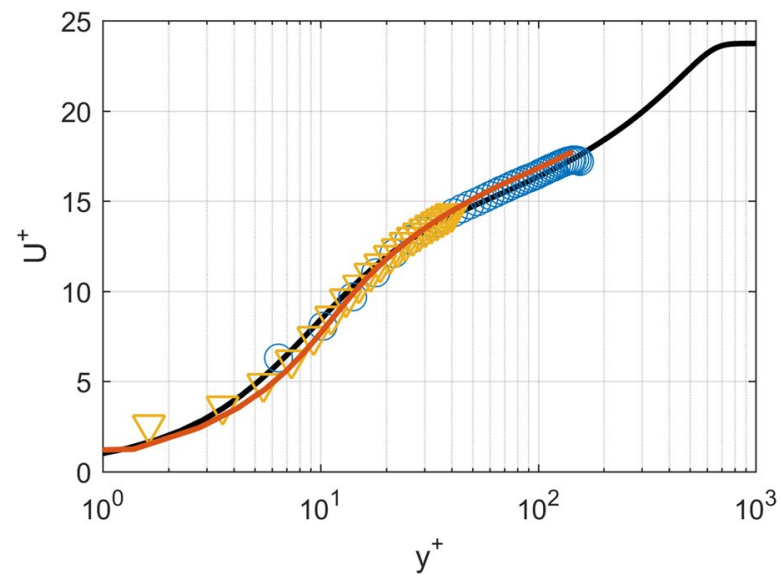

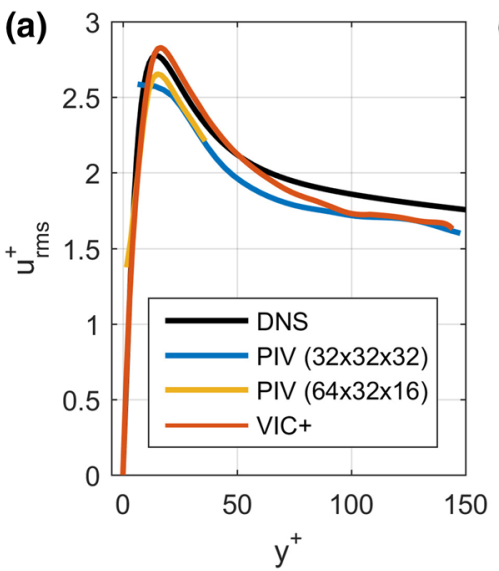
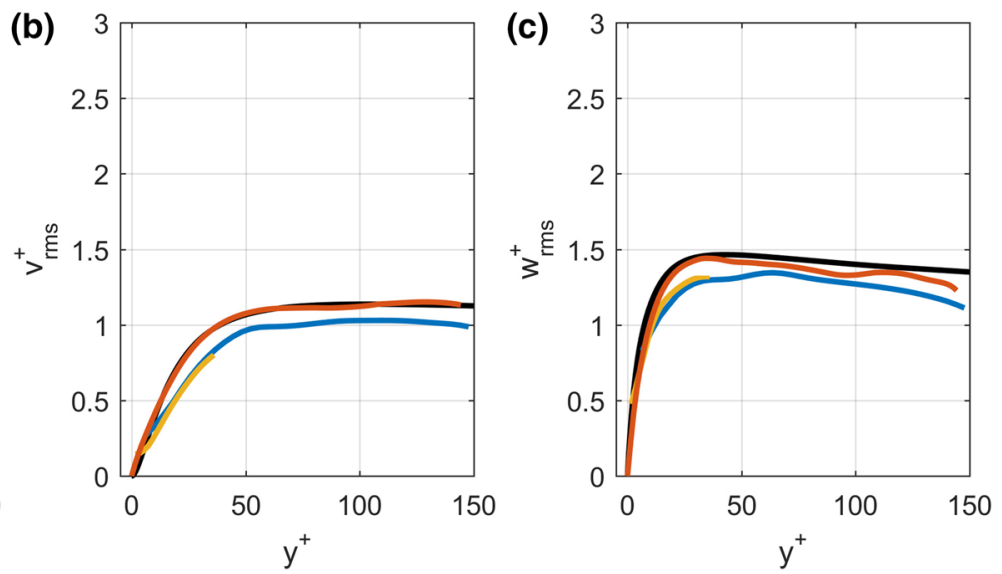

Fig. 4 Wall-normal profiles of rms velocity fluctuations returned by tomographic PIV with cubic and elongated correlation volumes and with VIC+, in comparison to the DNS result by Schlatter and Örlü (2010). Streamwise (a), wall-normal (b) and spanwise (c) velocity components

coefficient between the two components plotted in Fig. 5, $-\partial u / \partial x$ and $(\partial v / \partial y+\partial w / \partial z)$ is a commonly used technique to assess the measurement quality (Tsinober et al. 1992; Casey et al. 2013; Ganapathisubramani et al. 2007; amongst others). For the present tomographic PIV result, this cross-correlation coefficient equals 0.88 (Jodai and Elsinga 2016) and for the VIC+ result it is 0.9994 . Additionally, the rms divergence error is evaluated as the rms value of the velocity divergence between $y^{+}=40$ and $y^{+}=60$. It equals $0.013 u_{\tau}^{2} / v$ for PIV and $0.002 u_{\tau}^{2} / v$ for VIC + . This confirms that the latter yields, to a large extent, a divergence-free result.

\subsection{Instantaneous flow organization}

The pattern of coherent structures in the measurement volume at a single time instant is visualized in Fig. 6. Isosurfaces of vorticity magnitude $\left(\left|\omega^{+}\right|=0.3\right.$, red, shaded by wall-normal distance for clarity) are representative of vortex filaments mostly exhibiting arc, cane and hairpin shapes. The streamwise velocity (blue, $u^{+}=14$ ) instead highlights the organization of alternating elongated regions with momentum excess and defect. From visual inspection of the two data sets, a richer pattern of vortical structures is retrieved with the VIC+ reconstruction. Two hairpin vortices, $\mathrm{S} 1$ and $\mathrm{S} 2$, are indicated by arrows in Fig. 6. The largest of the two, $\mathrm{S} 1$, is visible in both the tomographic PIV and VIC+ reconstructions. On the other hand, at the selected vorticity level, S2 is clearly visible only within the VIC+ reconstruction. Also two tongue-like appendices (Zhou et al. 1999) are revealed attached to the hairpin structure. The vortex structures S1 and S2 are inspected in more detail in the paragraphs below.

Figure 7 illustrates the detailed view of the hairpin structure S1, as reconstructed by tomographic PIV (a) and VIC+ (b). The in-plane velocity vectors are plotted in the plane $x^{+}=-35$. Visual comparison shows no significant difference between the velocity vectors. Despite that streamwise vortex S3 is only marginally visible by the isosurface visualization in the PIV reconstruction, the 
Fig. 5 Joint pdf of $-\partial u / \partial x$ and $(\partial v / \partial y+\partial w / \partial z)$ evaluated between $y^{+}=40$ and 60 . The contours are plotted in logscale. Tomographic PIV (left) and VIC+ (right)

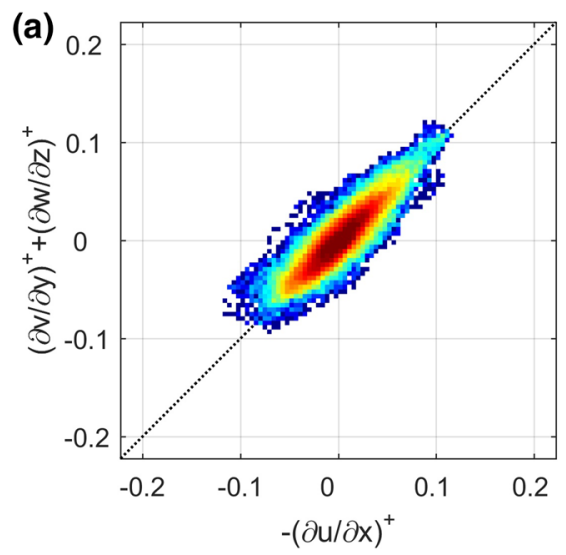

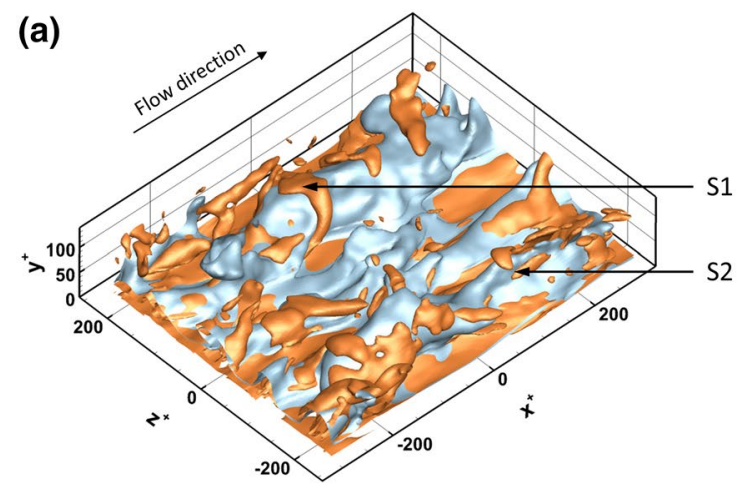

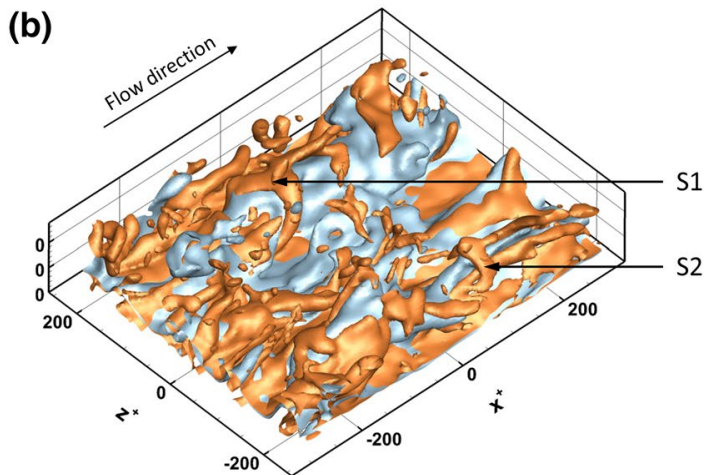

Fig. 6 Instantaneous flow organization in the measurement volume visualized by isosurfaces of vorticity magnitude $\left(\left|\omega^{+}\right|=0.3\right.$, orange $)$ and streamwise velocity $\left(u^{+}=14\right.$, blue $)$. Tomographic PIV (a) and VIC+ (b)
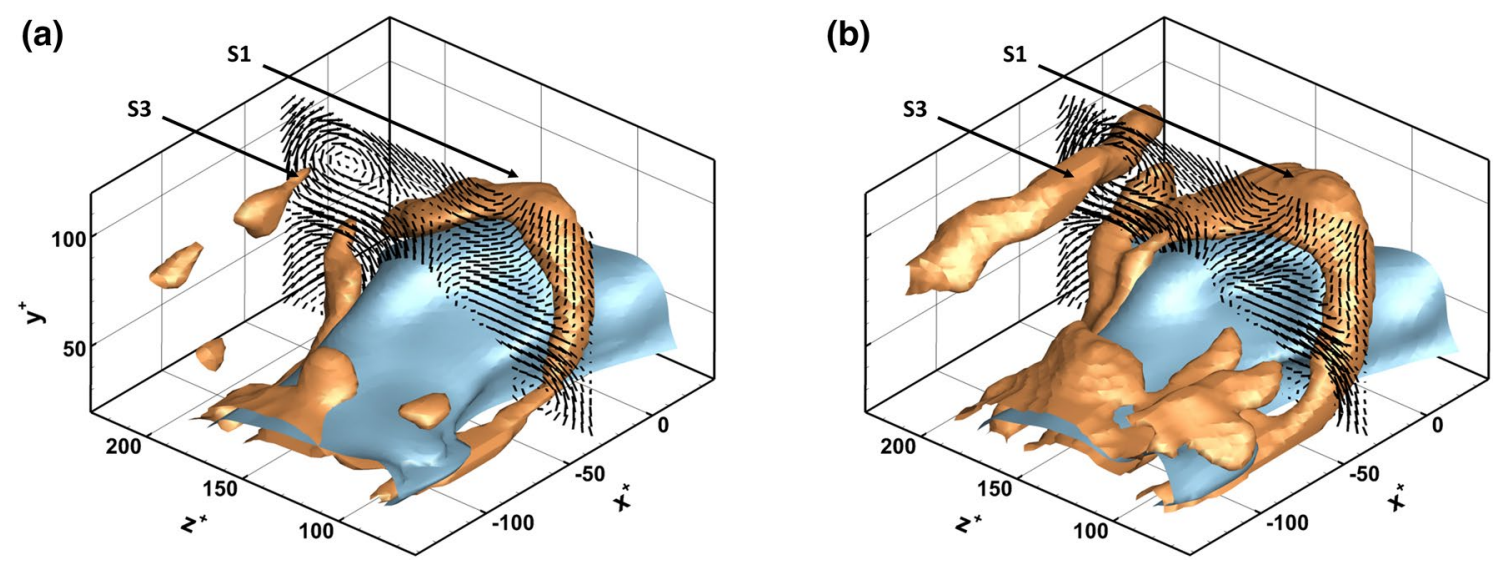

Fig. 7 Cut-out of hairpin structure $\mathrm{S} 1$, with the instantaneous in-plane velocity at $x^{+}=-35$. Isosurfaces show vorticity magnitude $\left(\left|\omega^{+}\right|=0.35\right.$, orange) and streamwise velocity $\left(u^{+}=12\right.$, blue). Tomographic PIV (a) and VIC+ (b)

velocity field does reveal the presence of the streamwise vortex. This indicates that the structure is present in both PIV and VIC+ results, but its associated vorticity magnitude is higher in the VIC+ result.
The question whether the PIV results are essentially similar to those produced by VIC+ except for a scaling factor in vorticity is addressed by analysing the vorticity topology at several set-values of the isosurface. Figure 8a-c illustrates the vorticity magnitude isosurface of the results 
obtained by tomographic PIV for a value ranging from $0.17 u_{\tau}^{2} / v$ to $0.3 u_{\tau}^{2} / v$. For the highest value, details of the vorticity pattern are revealed in the VIC+ data but appear significantly degraded in the PIV results. The same details become somehow more visible in the PIV result when a lower value for the isosurface is chosen. This difference in vorticity magnitude suggests that the effective spatial resolution is higher with VIC+ as compared to the tomographic PIV result.

The likeness in vortex structure shape is quantified by the cross-correlation coefficient between the PIV and VIC+ vorticity distributions. Specifically, we consider the streamwise vorticity component in the wall parallel plane at $y^{+}=100$. This yields a cross-correlation coefficient of 0.83 , which indicates that the vorticity distribution is very similar between the methods apart from a scaling constant. It supports the earlier conclusion obtained from vorticity visualizations.

The instantaneous flow organization is also studied by comparison of the $Q$-criterion (Hunt et al. 1988). Movies are added as supplementary material with the manuscript, showing the temporal evolution of the vortices visualized by the $Q$-criterion at three isosurface threshold levels; $Q^{+}$ $=3.5 \times 10^{-3}$ (movie 1$), Q^{+}=7 \times 10^{-3}$ (movie 2 ) and $Q^{+}=$ $14 \times 10^{-3}$ (movie 3 ). An extract of the instantaneous flow organization at time $t=374 \mathrm{~ms}$ is given in Fig. 9. The top row shows the results from tomographic PIV analysis and the bottom row shows the VIC+ result. From left to right, the results are plotted at the aforementioned thresholds of $Q$.

At each isosurface level of $Q$, the VIC+ result shows a richer pattern of vortical structures in comparison to tomographic PIV, which is consistent with the earlier observations made upon inspection of the isosurfaces of intense vorticity. Because the $Q$-criterion is calculated from squared velocity gradients, similarly to the dissipation rate (Sect. 4.4), it is more sensitive to spatial resolution as compared to vorticity, which linearly depends on velocity gradients. Therefore, $Q$ is expected to show more pronounced differences between tomographic PIV and VIC+. The vortical structure indicated by the black arrow, for example, is visible in the VIC+ result at the highest level of $Q$ (Fig. 9 bottom-right), but is not reconstructed by tomographic PIV at the lowest level of $Q$ (Fig. 9 top-left).

\subsection{Vorticity statistics}

The analysis in the previous sections suggests that the VIC+ data evaluation returns increased vorticity magnitude within the vortices and, therefore, has the potential to restore the original amplitude of vorticity fluctuations. In this section, the differences in vorticity magnitude are quantified using the rms vorticity fluctuations, which are presented in Fig. 10. The blue and yellow lines show the results obtained by tomographic PIV with, respectively, cubic and elongated interrogation volumes. The red lines are for VIC+, while the black lines show the DNS result (Schlatter and Örlü 2010) for reference.

The rms vorticity fluctuations (Fig. 10) by tomographic PIV are approximately $40 \%$ lower than the reference DNS level for $y^{+}>30$. This applies to all vorticity components. In the near-wall region $\left(y^{+}<30\right)$, a further reduction is observed with the cubic interrogation volumes, in comparison to the elongated volumes. Furthermore, the cubic interrogation volumes do not capture the peak in the rms wall-normal vorticity at $y^{+}=15$. For comparison, a $35 \%$ reduction in rms vorticity is expected for the present PIV interrogation window size according to Saikrishnan et al. (2006), who assessed the effect of spatial filtering. The reduced vorticity fluctuations by tomographic PIV in the current experiment can, therefore, be largely ascribed to the effects of spatial filtering of the velocity field.

The red lines in Fig. 10 show the VIC+ result. For all three components of vorticity, an increase in the level of rms fluctuations can be observed in comparison to tomographic PIV, which is consistent with the visualizations shown in Sect. 4.2. The vorticity fluctuations in all directions (Fig. 10) are found within 10\% of the reference from DNS for $y^{+}>20$. In the near-wall region $\left(y^{+}<20\right)$, on the other hand, there is a larger deviation with the DNS even
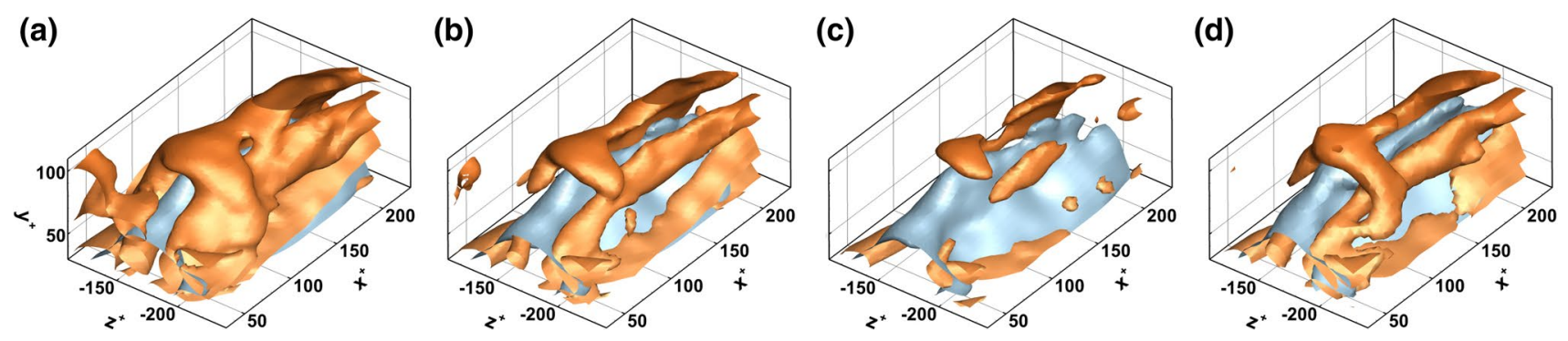

Fig. 8 Cut-out of hairpin structure S2. Isosurfaces of vorticity magnitude (orange) and streamwise velocity $\left(u^{+}=14\right.$, blue). a-c Tomographic PIV with, respectively, isosurfaces of vorticity magnitude at $\left|\omega^{+}\right|=0.17,0.23$ and 0.3 . d VIC + result with $\left|\omega^{+}\right|=0.3$ 

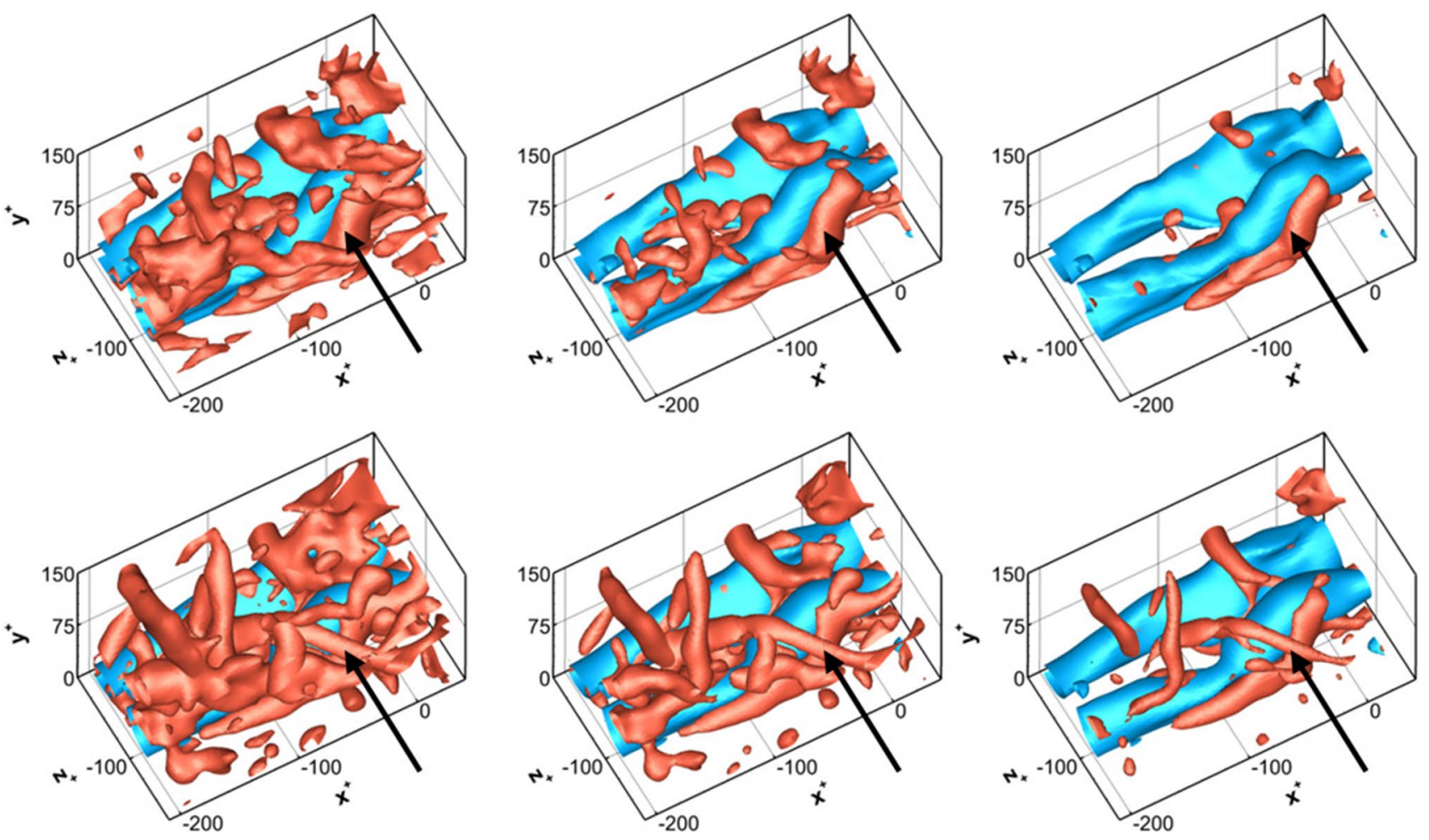

Fig. 9 Extract of the instantaneous flow organization at $t=374 \mathrm{~ms}$ visualized by isosurfaces of $u^{+}=-2.3$ (blue) and from left to right $Q^{+}=3.5 \times 10^{-3}, 7 \times 10^{-3}$, and $14 \times 10^{-3}$ (red isosurfaces). The top

row shows the results obtained by tomographic PIV and the bottom row the result of VIC+
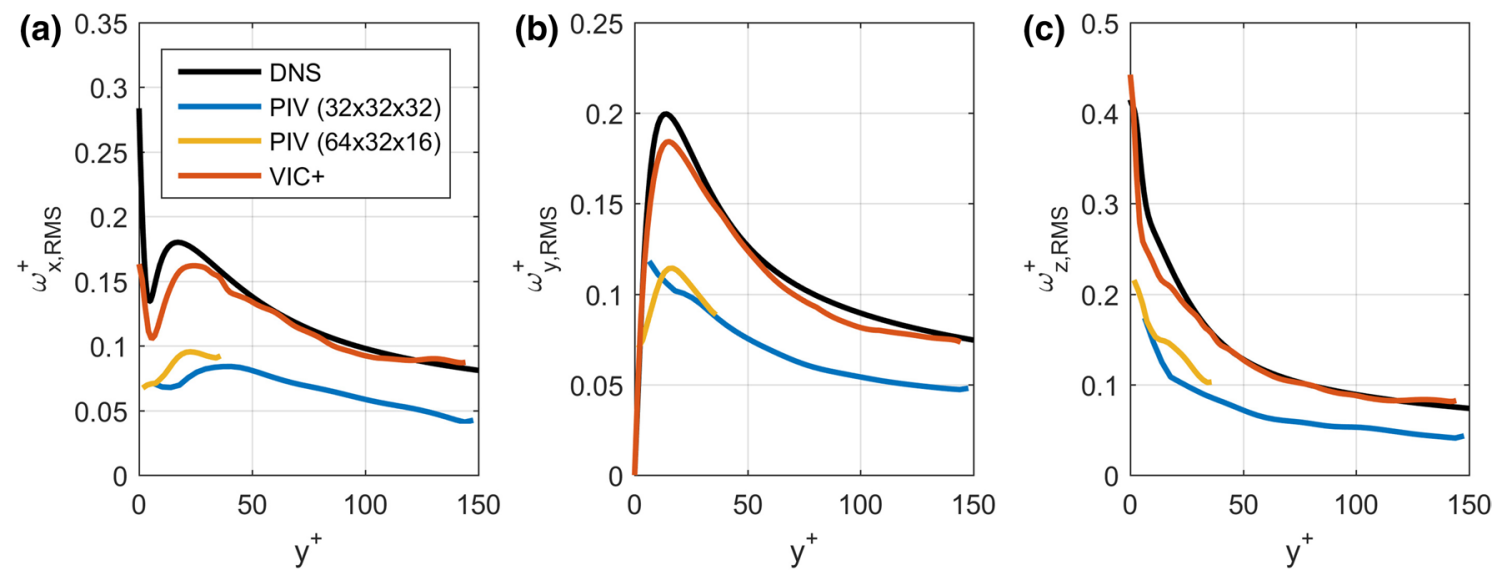

Fig. 10 Wall-normal profiles of rms vorticity returned by tomographic PIV with cubic and elongated correlation volumes and with VIC+, in comparison to the DNS result by Schlatter and Örlü (2010). Streamwise (a), wall-normal (b) and spanwise (c) rms vorticity fluctuations

with the VIC+ method, which is attributed to difficulties in resolving the strong velocity gradient in this region near the wall $\left(y^{+}<25\right)$ at a reduced density of identified particle tracks (c.f. Fig. 2). The wall-normal vorticity fluctuations (Fig. 10b), however, approach zero at the wall, and remain approximately within 10\% from the DNS reference along the full profile. The data in Saikrishnan et al. (2006) show that approximately $90 \%$ of the vorticity rms is captured at a spatial resolution of around 10-12 wall units. Based on this, the effective spatial resolution of VIC + in the present experiment is estimated at $<12$ wall units in all directions.

To assess the size of the coherent fine-scale eddies in the flow, the autocorrelation of the streamwise vorticity component is computed and plotted in Fig. 11 for two wall-normal 
locations. Streamwise vorticity is associated with the streamwise vortices in the near wall region. Based on the $-3 \mathrm{~dB}$ cutoff point $\left(R_{\omega, \omega}=0.5\right)$, the PIV results yield a vortex core size of approximately 16 wall units in spanwise direction. The core size appears insensitive to the wall-normal distance, which is consistent with the constant interrogation window size and the slowly varying vortex radius in turbulent boundary layers (Herpin et al. 2013). Inspecting Fig. 11, the VIC+ result shows a smaller vortex core size of 11 wall units. As shown in the Appendix, approximately a factor two smaller cutoff wavelength is expected for VIC+ in comparison to tomographic PIV. The present improvement in vortex core size is less, which suggests that the vortex size returned by VIC + is determined by the actual vortices in the flow and is not limited by the spatial resolution. This, moreover, is consistent with the rms vorticity fluctuation being nearly resolved by VIC+ (to with $10 \%$ of the DNS). For a channel flow at comparable Reynolds number $\left(R e_{\tau}=800\right)$, Tanahashi et al. (2004) report that the diameter of the coherent fine-scale eddies at these nearwall locations is approximately 8-9 times the Kolmogorov length scale, where the Kolmogorov length is estimated at 2.5 wall units (Stanislas et al. 2008). This corresponds to a typical radius of the vortical structures of 10-11 wall units, which matches the result obtained by VIC+. It is a further indication that the vortices are resolved. Based on the autocorrelations and the expected coherent fine-scale eddy size, the spatial resolution of the VIC+ method in the present experiment is conservatively estimated to be better than 11 wall units. This compares well to the abovementioned estimated spatial resolution based on the damping of the rms vorticity statistics.

As a consistency check, the VIC + velocity fields are filtered using a filter approximating the tomographic PIV processing chain used in Jodai and Elsinga (2016). The cross-correlation procedure is approximated using a moving average filter with a 15 wall units volume size corresponding to the interrogation volume size and subsequent polynomial filtering is using a 23 wall units moving average filter applied to the velocity gradient. After filtering the autocorrelation of the streamwise vorticity is evaluated, which is included in Fig. 11 (dashed line). The results closely resemble the PIV result. It again suggests that the VIC+ results approximate the actual flow and that the differences with respect to the tomographic PIV can be understood by the effect of its spatial filter. Moreover, the cross-correlation coefficient between the streamwise vorticity distribution obtained by the filtered VIC+ and PIV is 0.96 at $y^{+}=100$, which also shows that the filtered VIC + is nearly identical to the tomographic PIV result.

\subsection{Dissipation rate}

Compared to vorticity, the dissipation rate estimation is even more sensitive to spatial resolution, because it depends on velocity gradients raised to the power two. The underestimation of the dissipation rate by tomographic PIV is a problem recognized in recent literature (Tokgoz et al. 2012) and sub-grid scale modelling approaches have been proposed to improve the estimation of the dissipation rate from PIV (Sheng et al. 2000; Bertents et al. 2015). Following the results in the previous section, an improved dissipation rate estimate is expected with $\mathrm{VIC}+$, in comparison to tomographic PIV. To assess this conjecture, the wallnormal profile of the turbulent kinetic energy dissipation rate is plotted in Fig. 12. The blue and yellow lines show the results obtained by tomographic PIV with, respectively, cubic and elongated interrogation volumes. The red line shows the VIC+ result. For comparison, the DNS reference is plotted in black.

For the range $40<y^{+}<140$, the tomographic PIV result with cubic interrogation volumes underestimates the dissipation rate by $50 \%$. For $y^{+}<40$, the result of PIV with elongated interrogation volumes follows more accurately the trend of the DNS reference, but the result remains damped by $50 \%$ until $y^{+}=15$. Closer to the wall
Fig. 11 Normalized autocorrelation peak values of $x$-vorticity at $y^{+}=25($ left $)$ and $y^{+}=100$ (right)
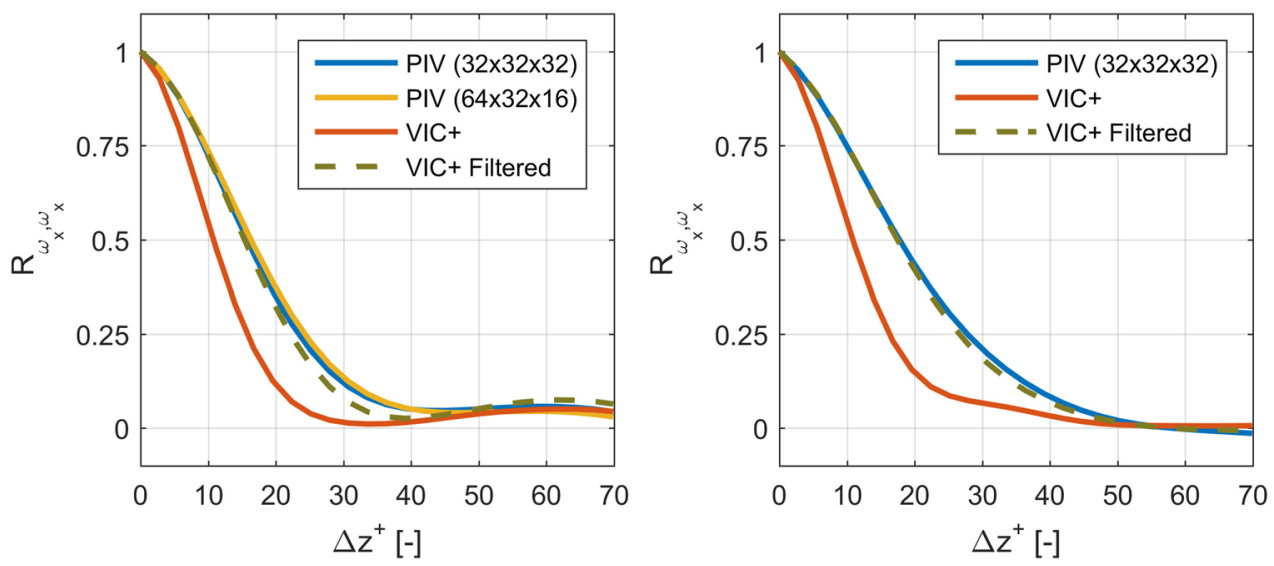


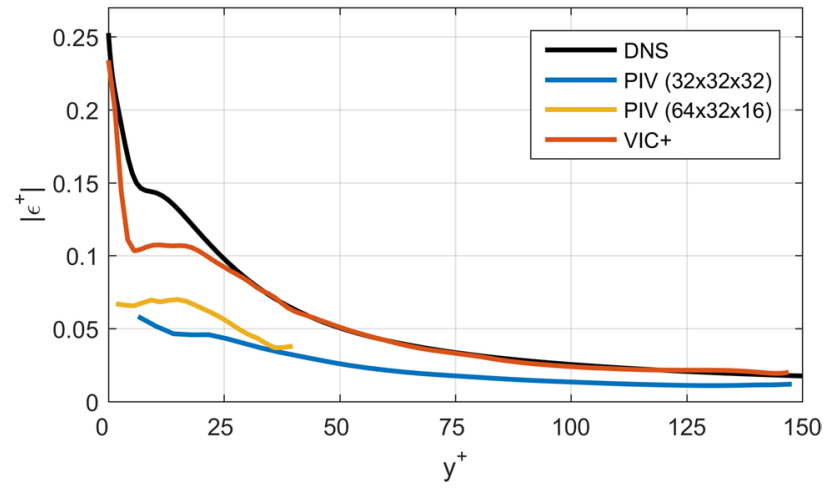

Fig. 12 Wall-normal profiles of kinetic energy dissipation rate returned by tomographic PIV with cubic and elongated correlation volumes and with VIC+, in comparison to the DNS result by Schlatter and Örlü (2010)

the dissipation predicted by PIV remains constant and the near-wall peak value is not captured. The strong damping of the dissipation confirms the difficulties encountered in the abovementioned literature when estimating dissipation from PIV measurements. The VIC+ method yields, on the other hand, a result within $5 \%$ of the reference for $y^{+}>25$, with no need to introduce further sub-grid scale models. Closer to the wall, the damping increases and becomes $20 \%$ at $y^{+}=15$. Moreover, the VIC+ result shows a peak dissipation value at the wall within $8 \%$ of the reference value.

\subsection{Practical aspects and computational cost}

The present section discusses some practical aspects and computational costs of the VIC+ technique in comparison to tomographic PIV. Contrary to tomographic PIV, which can be used for low repetition rate dual-pulse measurements, the VIC+ technique can be applied only when timeresolved Lagrangian particle tracking measurements (e.g. tomographic PTV or Shake-the-Box) are available. The latter are required to provide measurements of both instantaneous tracer particle velocity and its material derivative. When only flow statistics are desired, as in the present manuscript, the requirement for time-resolved measurements can lead to a large measurement dataset and consequentially a large computational burden. The latter was alleviated in the present study by processing short bursts, each separated by approximately a large-scale turnover time $\left(\delta_{99} / u_{\infty}\right)$ to obtain the statistics. To further reduce storage requirements, the measurement data can also be acquired in bursts to allow for time-resolved particle tracking. With this processing, the computer memory requirements for tomographic PIV and VIC+ are found to be on the same order of magnitude, but the computation time for
VIC+ is approximately an order of magnitude longer than for tomographic PIV.

When a full time-series is to be processed for inspection of the flow temporal evolution, however, the computation time required for the iterative VIC+ procedure can be reduced to the level of tomographic PIV cross-correlation. This is done by starting the VIC+ procedure at each time instant with an estimate of the velocity field based on the result from the previous time instant. The estimated can, for instance, be obtained from a short time advancement of the flow using VIC (Schneiders et al. 2014). This is analogous to the sliding implementation of motion tracking enhanced MART (MTE, Novara et al. 2010) by sequential MTE (SMTE, Lynch and Scarano 2015). The supplementary material shows movies of the $Q$-Criterion of $1173 \mathrm{~ms}$ of flow obtained using this procedure.

\section{Conclusions}

The VIC+ method was applied to an actual turbulent boundary layer experiment at $R e_{\theta}=2038$, and was demonstrated to resolve the rms vorticity fluctuations to within $10 \%$ of a reference from DNS. In comparison, tomographic PIV analysis yielded approximately $40 \%$ damping of the vorticity fluctuations. The VIC+ flow fields comply with the continuity equation and they are consistent with the measured velocity and acceleration of the tracer particles. Additionally, the present vorticity and dissipation statistics match an existing DNS reference to within 5\% for $y^{+}>25$, which suggests that the VIC+ flow fields are well resolved and accurate. In comparison, dissipation statistics are damped by $50 \%$ by tomographic PIV. The novel aspect is that the dissipation statistics are obtained from the measurement without relying on isotropy assumptions or correction factors. The effective spatial resolution of VIC+ in the present experiment was conservatively estimated at 11 wall units. Isosurface visualizations of instantaneous velocity and vorticity showed increased coherence in the vortical structures at higher isosurface levels. Tomographic PIV, however, revealed similar vortical structures at lower vorticity magnitude. The study demonstrates that the VIC+ method can be applied effectively to actual tomographic PIV and volumetric particle tracking measurements for increased reconstruction quality of vorticity and dissipation. The study is supported by quantification of the spatial response of VIC+ with a sine-wave lattice analysis. The results indicate a twofold increase of spatial resolution with respect to cross-correlation interrogation.

Acknowledgements This research is partly funded by LaVision $\mathrm{GmbH}$. 
Open Access This article is distributed under the terms of the Creative Commons Attribution 4.0 International License (http:// creativecommons.org/licenses/by/4.0/), which permits unrestricted use, distribution, and reproduction in any medium, provided you give appropriate credit to the original author(s) and the source, provide a link to the Creative Commons license, and indicate if changes were made.

\section{Appendix: Spatial response of the VIC+ interpolation technique}

The spatial response of the $\mathrm{VIC}+$ interpolation technique is assessed in this appendix and compared to the response of multi-pass tomographic PIV. The approach taken is similar to that by Scarano and Riethmuller (2000) and Schrijer and Scarano (2008), who assessed the modulation of PIV crosscorrelation techniques using analytical sine and cosine flow fields with a predefined wavelength. Because the $\mathrm{VIC}+$ method leverages particle acceleration measurements for the dense interpolation of velocity to a grid, the present assessment considers a velocity field where the velocity material derivative is non-zero and non-homogeneous:

$u=\left\{\begin{array}{l}u=A \sin \frac{2 \pi x}{\Lambda} \sin \frac{2 \pi y}{\Lambda} \\ v=A \cos \frac{2 \pi x}{\Lambda} \cos \frac{2 \pi y}{\Lambda} \\ w=0\end{array}\right.$

Tomographic PIV and PTV measurements are simulated in a volume of $L_{x} \times L_{y} \times L_{z}=500 \times 500 \times 500$ voxels, with Gaussian-shaped tracer particles of 2 voxels diameter and a uniform peak intensity value. The tracer particle concentration is $C=5 \times 10^{-5}$ particles per voxel (ppv) and the wavelength of the waves, $\Lambda$, is varied between 20 and 500 voxels. No particle reconstruction errors are simulated for the reconstructed volumes and the PTV measurements to assess the ideal response for both cases. The maximum tracer particle displacement is equal to $A=2$ voxels. For tomographic PIV analysis, a multi-pass volume deformation algorithm is used with 8 passes. After each pass, outliers are removed using universal outlier detection (Westerweel and Scarano 2005) and the vector field is smoothed using Gaussian smoothing with a $3 \times 3 \times 3$ vector kernel. No smoothing is applied on the final pass. The interrogation volume (IV) size is chosen such that there are on average five particles present in a volume and $75 \%$ overlap is used. Five tracer particles are considered the minimum number for reliable cross-correlation peak detection. Therefore, the present interrogation volume size represents the best possible spatial resolution for tomographic PIV. This yields a volume size of $48 \times 48 \times 48$ voxels and a vector spacing of 12 voxels. For VIC + analysis, the grid spacing, $h$, is determined using $h=0.25 C^{-1 / 3}$ (Schneiders and Scarano 2016). This yields a vector spacing of 7 voxels.

Recent literature reports that the spatial response of iterative cross-correlation with window deformation in the above-mentioned flow case improves slightly upon the squared sinc function (Schrijer and Scarano 2008). This is confirmed in Fig. 13, which shows the amplitude modulation of cross-correlation (blue line) and the squared sinc function (black line) plotted against the normalized interrogation volume size $l^{*}=\mathrm{IV} / \Lambda$. The amplitude modulation $u / u_{0}$ is defined as the ratio of peak streamwise velocity in the measurement volume in comparison to the reference provided by Eq. (4). The peak velocity is evaluated from the tomographic PIV and VIC+measurements grids through second-order polynomial interpolation. The $-3 \mathrm{~dB}$ cutoff wavelength for PIV equals $l_{\mathrm{c}}^{*}=0.53$ $\left(r_{\mathrm{c}}^{*}=0.17\right)$ and for the sinc function $l_{\mathrm{c}}^{*}=0.44\left(r_{\mathrm{c}}^{*}=0.14\right)$, which corresponds well to the values reported by Schrijer and Scarano (2008). Because VIC+does not use interrogation volumes, the normalized minimum interparticle distance $r^{*}=\bar{r} / \Lambda$ is a more appropriate scaling

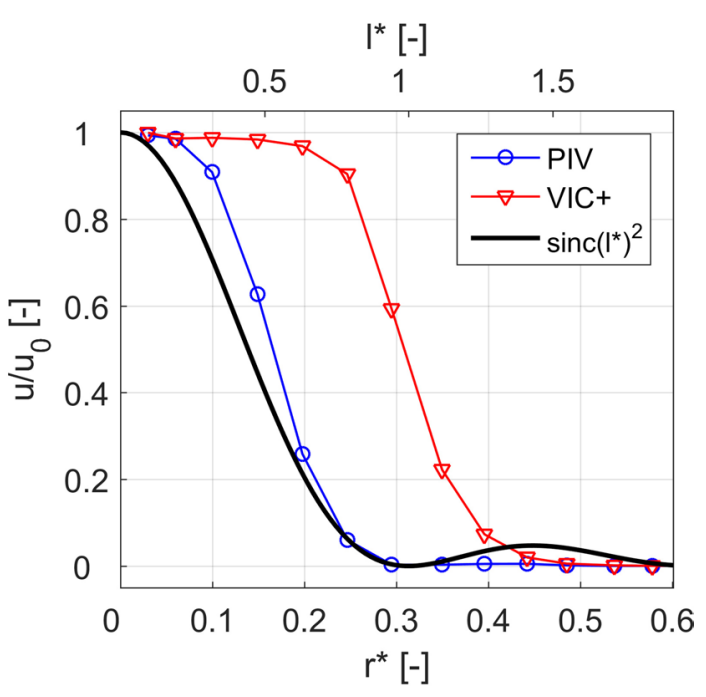

Fig. 13 Modulation flow fields by cross-correlation (blue line) and VIC+ (red line), as a function of wavelength. For comparison, also the squared sinc function is plotted (black line)

Table 2 Normalized window size $l^{*}$ and normalized inter-particle distance $r^{*}$ at the $-3 \mathrm{~dB}$ cutoff point for the squared $\sin c$ function, PIV cross-correlation analysis and VIC+

\begin{tabular}{llll}
\hline & $\sin c^{2}$ & PIV & VIC+ \\
\hline$l_{\mathrm{c}}^{*}$ & 0.44 & 0.53 & 0.98 \\
$r_{\mathrm{c}}^{*}$ & 0.14 & 0.17 & 0.31 \\
\hline
\end{tabular}


Fig. 14 Streamwise velocity contour plot at the center $z$-plane at a normalized particle separation of $r^{*}=0.2$ and a normalized window size of $l^{*}$ $=1$
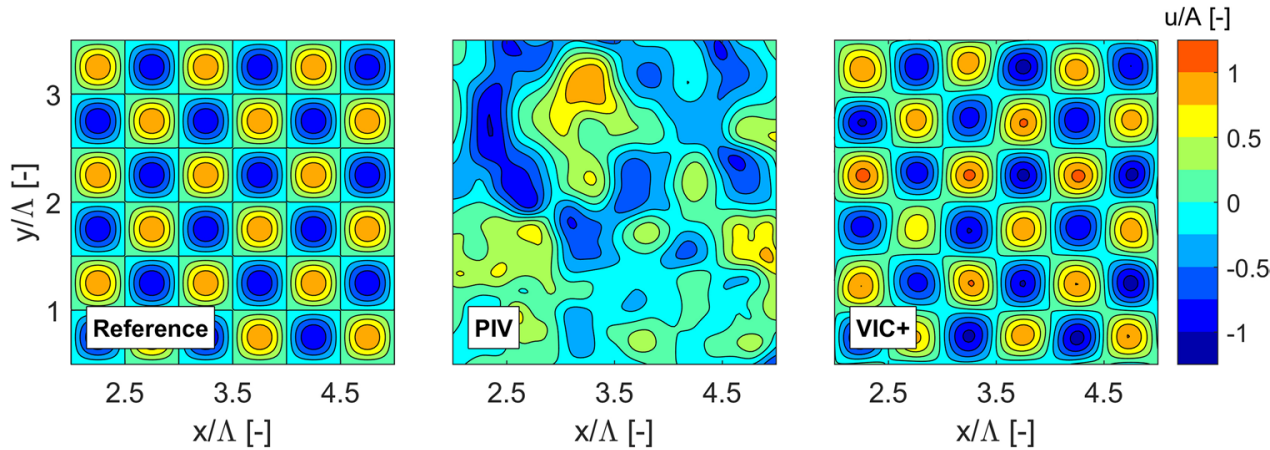

for comparison of the results and is, therefore, used on the bottom horizontal axis. The VIC + technique shows a practically flat response up to $r^{*}=0.2$ and a $-3 \mathrm{~dB}$ cutoff wavelength of $r_{\mathrm{c}}^{*}=0.31$, which is approximately twice the cutoff wavelength of PIV, as summarized in Table 2.

For illustration of the results, Fig. 14 shows the streamwise velocity component at the center z-plane for a normalized average minimum inter-particle separation of $r^{*}=0.2$. The reference flow field (left figure) shows the expected pattern of sine waves resulting from Eq. (4). Using crosscorrelation analysis yields a significantly modulated flow field $\left(u / u_{0}=0.29\right)$, but on the other hand the VIC+ technique is still able to find the reference flow field with peak values within $10 \%$ of the reference.

\section{References}

Agüera N, Cafiero G, Astarita T, Discetti S (2016) Ensemgle 3D PTV for high resolution turbulent statistics. Meas Sci Technol 27:124011

Atkinson C, Coudert S, Foucaut JM, Stanislas M, Soria J (2011) The accuracy of tomographic particle image velocimetry for measurements of a turbulent boundary layer. Exp Fluids 50:1031-1056

Bertents G, van der Voort D, Bocenagra-Evans H, van de Water W (2015) Large-eddy estimate of the turbulent dissipation rate using PIV. Exp Fluids 56:89

Casey TA, Sakakibara J, Thoroddsen ST (2013) Scanning tomographic particle image velocimetry applied to a turbulent jet. Phys Fluids 25:025102

de Silva MC, Philip J, Marusic I (2013) Minimization of divergence error in volumetric velocity measurements and implications for turbulence statistics. Exp Fluids 54:1557

Elsinga GE, Marusic I (2010) Evolution and lifetimes of flow topology in a turbulent boundary layer. Phys Fluids 22:015102

Elsinga GE, Scarano F, Wieneke B, van Oudheusden BW (2006) Tomographic particle image velocimetry. Exp Fluids 41:933-947

Elsinga GE, Westerweel J, Scarano F, Novara M (2011) On the velocity of ghost particles and the bias errors in Tomographic-PIV. Exp Fluids 50:825-838

Elsinga GE, Poelma C, Schröder A, Geisler R, Scarano F, Westerweel J (2012) Tracking of vortices in a turbulent boundary layer. J Fluid Mech 697:273-295
Ganapathisubramani B, Lakshminarasimhan K, Clemens NT (2007) Determination of complete velocity gradient tensor by using cinematographic stereoscopic PIV in a turbulent jet. Exp Fluids 42:923-939

Gesemann S, Huhn F, Schanz D, Schröder A (2016) From noisy particle tracks to velocity, acceleration and pressure fields using B-splines and penalties. In: 18th Int. Symp. on Applications of Laser and Imaging Techniques to Fluid Mechanics. Lisbon, Portugal. 4-7 July

Herpin S, Stanislas M, Foucaut JM, Coudert S (2013) Influence of the Reynolds number on the vortical structures in the logarithmic region of turbulent boundary layers. J Fluid Mech 716:5-50

Hunt J, Wray A, Moin P (1988) Eddies, stream, and convergence zones in turbulent flows. Center for Turbulence Research Report CTR-S88, pp 193-208.

Jodai Y, Elsinga GE (2016) Experimental observation of hairpin autogeneration events in a turbulent boundary layer. J Fluid Mech 795:611-633

Kähler CJ, Scharnowski S, Cierpka C (2012) On the uncertainty of digital PIV and PTV near walls. Exp Fluids 52:1641-1656

Lynch K, Scarano F (2015) An efficient and accurate approach to MTE-MART for time-resolved tomographic PIV. Exp Fluids $56: 66$

Lynch K, Pröbsting S, Scarano F (2014). Temporal resolution of timeresolved tomographic PIV in turbulent boundary layers. In: 17th international symposium on the application of laser techniques to fluid mechanics. Lisbon, Portugal

Malik NA, Dracos T, Papantoniou DA (1993) Particle tracking velocimetry in three-dimensional flows. Exp Fluids 15:279-294

Marusic I, McKeon BJ, Monkewitz PA, Nagib HM, Smits AJ, Sreenivasan KR (2010) Wall-bounded turbulent flows at high Reynolds numbers: Recent advances and key issues. Phys Fluids 22(065103): $1-24$

Novara M, Scarano F (2013) A particle-tracking approach for accurate material derivative measurements with tomographic PIV. Exp Fluids 54:1-12

Novara M, Batenburg KJ, Scarano F (2010) Motion trackingenhanced MART for tomographic PIV. Meas Sci Technol 21:035401

Saikrishnan N, Marusic I, Longmire EK (2006) Assessment of dual plane PIV measurements in wall turbulence using DNS data. Exp Fluids 41:265-278

Scarano F, Riethmuller ML (2000) Advances in iterative multigrid PIV image processing. Exp Fluids 29(Supplement 1):S051-S060.

Schanz D, Gesemann S, Schröder A (2016) Shake-The-Box: Lagrangian particle tracking at high particle image densities. Exp Fluids 57:70

Schlatter P, Örlü R (2010) Assessment of direct numerical simulation data of turbulent boundary layers. J Fluid Mech 659:116-126 
Schneiders JFG, Scarano F (2016) Dense velocity reconstruction from tomographic ptv with material derivatives. Exp Fluids 57:139

Schneiders JFG, Dwight RP, Scarano F (2014) Time-supersampling of 3D-PIV measurements with vortex-in-cell simulation. Exp Fluids 55:1692

Schneiders JFG, Dwight RP, Scarano F, (2015) Tomographic PIV noise reduction by simulating repeated measurements. In: 11th International Symposium on Particle Image Velocimetry, PIV15, Santa Barbara, CA, USA, September 14-16

Schneiders JFG, Pröbsting S, Dwight RP, van Oudheusden BW, Scarano F (2016) Pressure estimation from single-snapshot tomographic PIV in a turbulent boundary layer. Exp Fluids 57:53

Schrijer FFJ, Scarano F (2008) Effect of predictor-corrector filtering on the stability and spatial resolution of iterative PIV interrogation. Exp Fluids 45:927-941

Schröder A, Geisler R, Elsinga GE, Scarano F, Dierksheide U (2008) Investigation of a turbulent spot and a tripped turbulent boundary layer flow using time-resolved tomographic PIV. Exp Fluids 44:305-316

Schröder A, Geisler R, Staack K, Elsinga GE, Scarano F, Wieneke B, Henning A, Poelma C, Westerweel J (2011) Eulerian and Lagrangian views of a turbulent boundary layer flow using timeresolved tomographic PIV. Exp Fluids 44:305-316

Sheng J, Meng H, Fox R (2000) A large eddy PIV method for turbulence dissipation rate estimation. Chem Eng Sci 55:4423-4434

Stanislas M, Perret L, Foucaut J-M (2008) Vortical structures in the turbulent boundary layer: a possible route to a universal representation. J Fluid Mech 602:327-382

Tanahashi M, Kang S-J, Miyamoto T, Shiokawa S, Miyauchi T (2004) Scaling law of fine scale eddies in turbulent channel flows up to $R e_{\tau}=800$. Int $\mathrm{J}$ Heat Fluid Flow 25:331-340
Tokgoz S, Elsinga GE, Delfos R, Westerweel J (2012) Spatial resolution and dissipation rate estimation in Taylor-Couette flow for tomographic PIV. Exp Fluids 53:561-583

Tsinober A, Kit E, Dracos T (1992) Experimental investigation of the field of velocity gradients in turbulent flows. J Fluid Mech 242:169-192

van Gent P, Michaelis D, van Oudheusden BW, Weiss P-É, de Kat R, Laskari A, Jeon YJ, David L, Schanz D, Huhn F, Gesemann S, Novara M, McPhaden C, Neeteson N, Rival D, Schneiders JFG, Schrijer F (2017) Comparative assessment of pressure field reconstructions from particle image velocimetry measurements and Lagrangian particle tracking. Exp Fluids. doi:10.1007/ s00348-017-2324-Z

Vedula P, Adrian R (2005) Optimal solenoidal interpolation of turbulent vector fields: Application to PTV and super-resolution PIV. Exp Fluids 39:213-221

Westerweel J, Scarano F (2005) Universal outlier detection for PIV data. Exp Fluids 39:1096-1100

Wieneke B (2013) Iterative reconstruction of volumetric particle distribution. Meas Sci Technol 24:024008

Zhong JL, Weng JY, Huang TS (1991) Vector field interpolation in fluid flow. Digital signal processing. In: International conference on DSP, Florence, Italy

Zhou J, Adrian RJ, Balachandar SK en dall TM (1999) Mechanisms for generating coherent packets of hairpin vortices in channel flow. J Fluid Mech 387:353-396 